\title{
On Analytic Functions with Divergent Series of Taylor Coefficients
}

\author{
Piotr Kot $^{1}$
}

Received: 30 August 2017 / Accepted: 24 October 2017 / Published online: 31 October 2017

(C) The Author(s) 2017. This article is an open access publication

\begin{abstract}
We consider a bounded, circular, strictly convex domain $\Omega$ with $C^{2}$ boundary. We show that there exists a holomorphic function $f_{1}$, continuous to the boundary such that every slice function has a series of Taylor coefficients divergent with every power $p \in[0,2)$. We also construct inner function $f_{2}$ which every slice is also inner and has series of Taylor coefficients with the same property. Next we generalize it to obtain $f_{3} \in \mathbb{O}(\Omega)$ with given modulus a.e. on all slices and Taylor series as above.
\end{abstract}

Keywords Inner function - Taylor series - Slice function - Boundary values of holomorphic function

Mathematics Subject Classification Primary 32A40; Secondary 32A05

\section{Introduction}

\subsection{Historical Background}

In $[8,7.2]$ Rudin gives some examples of boundary behavior of holomorphic functions in complex unit balls with dimensions 2 and 3. Ryll and Wojtaszczyk observed [9, Theorem 1.2 + Remark 1.10] that similar examples could be constructed in arbitrary dimension. The crucial tool used in the reminded constructions is [9, Theorem 1.2]: there exist polynomials $\left\{p_{n}\right\}$ homogeneous of degree $n$ on the unit ball $B^{d}$ such that

Communicated by Ahmed Sebbar.

$\triangle$ Piotr Kot

Piotr.Kot@agh.edu.pl

1 Faculty of Applied Mathematics, AGH University of Science and Technology,

Mickiewicz Avenue 30, 30-059 Cracow, Poland 


$$
\left\|p_{n}\right\|_{2}=1 \text { and }\left\|p_{n}\right\|_{\infty} \leq \frac{2^{d}}{\sqrt{\pi}} .
$$

This tool can be used to convert some one-dimensional examples into a multidimensional case. An interesting example of such an application is presented in the paper [10], where Wojtaszczyk generalizes the following one variable result:

- there exists a function $f(z)=\sum_{n=0}^{\infty} a_{n} z^{n}$ analytic for $|z|<1$ and continuous to the boundary such that $\sum_{n=0}^{\infty}\left|a_{n}\right|^{p}=\infty$ for all $p<2$

to a multi dimensional case.

\subsection{Motivations}

Our inspiration is the paper [10], where it was proven that there exists a function $f \in A\left(\mathbb{B}^{d}\right)$ such that almost every slice function of $f$ has a series of Taylor coefficients divergent with every power $p<2$.

We are going to strengthen the Wojtaszczyk's result [10] by showing that:

(1) the unit ball $\mathbb{B}^{d}$ can be replaced by $\Omega$ bounded, circular, strictly convex domain with $C^{2}$ boundary,

(2) almost every slice can be replaced by every slice,

(3) it is possible to construct $f \in A(\Omega)$ in the elementary way,

(4) it is possible to construct an inner function for whom every slice function is both inner and has a series of Taylor coefficients divergent.

(5) it is possible to construct a holomorphic function with a given modulus a.e. on slices with a Taylor series with the properties as given above.

Note that the first construction of an inner function for whom all slices functions are also inner was presented in [1] for the unit ball and in [6, Theorem 3.2] for bounded, circular, strictly convex domain $\Omega$ with $C^{2}$ boundary.

The tool like the one (1.1) is too weak to achieve (1)-(5). Therefore, we use a generalization of Ryll-Wojtaszczyk polynomials (see $[4,5,11]$ ). However, the results obtained in these papers have a wider applications when we combine them with information about holomorphic functions with bounded growth from the paper [3]. Such a combination was used before in [6, Theorem 3.2].

Wojtaszczyk used (1.1) and he also needed surjectivity of the operator $T: A\left(\mathbb{B}^{d}\right) \rightarrow$ $l_{2}$ defined by $T(f)=\left(\left\langle f, p_{2^{n}}\right\rangle\right)_{n=0}^{\infty}$, which was showed in [10, Proposition] (see also [7, Proposition 3.1]) by duality theorem. In one variable case, the constructive proof of such a surjectivity can be found in [2]. As it was mentioned by Wojtaszczyk, it would be interesting to have such a constructive proof in case of the unit ball $\mathbb{B}^{d}$. We do not know if a similar result can be obtained for different domains than $\mathbb{B}^{d}$.

Another crucial tool used in [10] was "scrambling lemma". This lemma needs unitary maps presented on the unit ball. A lack of mentioned surjectivity or "scrambling lemma" makes the propagation of Wojtaszczyk proof impossible. 


\subsection{Notations}

Let $\Omega$ be a bounded, circular, strictly convex domain with $C^{2}$ boundary.

For a given $f \in \mathbb{O}(\Omega)$ we study the slice function

$$
f_{z}: \mathbb{B}^{1}=\mathbb{D} \ni \lambda \rightarrow f(\lambda z) .
$$

We also define the middle value $\|f\|_{z}:=\sup _{r<1} \sqrt{\int_{0}^{1}\left|f\left(r e^{2 \pi i t} z\right)\right|^{2} d t}$ of holomorphic function $f$ on a circle given by the point $z \in \partial \Omega$. By $f^{*}$ we denote radial limit of $f$. It is known that $\|f\|_{z}<\infty$ implies that $f$ has such limit a.e on boundary of the slice $\mathbb{D} z$. A similar definition can be given for a function $g$ on $\partial \Omega$. Then, we define a slice function $g_{z}: \partial \mathbb{D} \ni \lambda \rightarrow g(\lambda z)$ and a norm of $g$ on slice: $\|g\|_{z}=\sqrt{\int_{0}^{1}\left|g\left(e^{2 \pi i t} z\right)\right|^{2} d t}$. We need the following facts: ${ }^{1}$

Theorem 1 [3, Theorem 3.2] There exists a natural number $N_{1}=N_{1}(\partial \Omega)$ such that, if $\varepsilon \in(0,1), T$ is a compact subset of $\Omega, h$ is a continuous, strictly positive function on $\partial \Omega$, then there exist holomorphic functions $f_{1}, \ldots, f_{N_{1}} \in A(\Omega)$ such that:

(1) $\left|f_{j}\right|<\varepsilon$ on $T$,

(2) $\left|f_{j}\right|<h$ on $\partial \Omega$,

(3) $\frac{1}{2} h<\max _{j=1, \ldots, N_{1}}\left|f_{j}\right|$ on $\partial \Omega$.

We can observe that it is possible to replace holomorphic functions in the above fact with the polynomials (see [6, Lemma 2.1]).

Theorem 2 [5, Theorem 2.5] There exists a natural number $N_{2}$ such that there exists $N_{3} \in \mathbb{N}$ such that for all integers $N$ and $m_{1}, \ldots, m_{N_{2}}$ with $N_{3} \leq N \leq m_{1} \leq$ $\cdots \leq m_{N_{2}} \leq 2 N$ there exist homogeneous polynomials $p_{m_{1}}, \ldots, p_{m_{N_{2}}}$ of a degree $m_{1}, \ldots, m_{N_{2}}$ respectively such that $\frac{1}{2}<\max _{i=1, \ldots, N_{2}}\left|p_{m_{i}}(z)\right|<1$ for all $z \in \partial \Omega$.

\subsection{Main Result}

We obtain the following fact (see Theorems 4, 5, 6):

Theorem There exists a holomorphic function $f \in A(\Omega)$ such that every slice function has a series of Taylor coefficients divergent with every power $s \in[0,2)$.

In fact our methods work also in case of an inner function:

Theorem There exists an inner function $f \in \mathbb{O}(\Omega)$ such that for all $z \in \partial \Omega$ we have the following properties:

- $\left|f_{z}^{*}\right|=1$ a.e. on $\partial \mathbb{D}$,

- $f_{z}$ has a series of Taylor coefficients divergent with every power $s \in[0,2)$.

The above result has the following generalization:

\footnotetext{
${ }^{1}$ Theorem [3, Theorem 3.2] and [5, Theorem 2.5] are proven in a more general situation than we need, so we simplify it here to satisfy our expectations.
} 
Theorem Let $\psi$ be a positive LSC function on $\partial \Omega$ for whom every slice function $\psi_{z}: \partial \mathbb{D} \ni \lambda \rightarrow \psi(\lambda z)$ is from $L^{2}$. There exists a holomorphic function $f \in \mathbb{O}(\Omega)$ such that for all $z \in \partial \Omega$ we have the following properties:

- $\left|f_{z}^{*}\right|=\psi_{z}$ a.e. on $\partial \mathbb{D}$,

- $f_{z}$ has a series of Taylor coefficients divergent with every power $s \in[0,2)$.

In all the above facts a similar method was used which is presented in Lemma 3.

To obtain Taylor series of a given function $f \in \mathbb{O}(\Omega)$ it is necessary to find homogeneous expansion for $f$ :

$$
f(z)=\sum_{n=0}^{\infty} p_{n}(z)
$$

where $p_{n}$ is a homogeneous polynomial of degree $n$. Now we have Taylor coefficients expansion for a slice function:

$$
f_{z}: \lambda \rightarrow f(\lambda z)=\sum_{n=0}^{\infty} p_{n}(z) \lambda^{n},
$$

so we can construct a holomorphic function $f \in \mathbb{O}(\Omega)$ whose every slice function $f_{z}$ is in $H^{2}$ so that:

$$
\sum_{n=0}^{\infty}\left|p_{n}(z)\right|^{s}=\infty
$$

for $s \in[0,2)$. Note that the $H^{2}$ property of $f_{z}$ implies:

$$
\sum_{n=0}^{\infty}\left|p_{n}(z)\right|^{2}<\infty
$$

for $z \in \partial \Omega$.

\section{Divergent Taylor Series}

Lemma 3 There exists a constant $\gamma>0$ such that for $k \in \mathbb{N}, a, \theta \in(0,1)$ and a continuous positive function $g$ on $\partial \Omega$ we can choose $N \in \mathbb{N}$ and polynomials $q_{1}, \ldots, q_{N}$ such that:

(1) each non zero monomial in $q_{i}$ has a degree greater than $k$ and smaller than any monomial's degree in $q_{i+1}$,

(2) $\left|q_{j}\right|<$ ag on $\partial \Omega$,

(3) $\left|\sum_{i=1}^{m} q_{i}\right|<$ on $\partial \Omega$ for $m \geq 1$, 
(4) $\left\|q_{m+1}\right\|_{z}^{2}>\gamma\left\|\min \left\{a g, g-\left|\sum_{i=1}^{m} q_{i}\right|\right\}\right\|_{z}^{2}$ for $z \in \partial \Omega$ and $m \geq 1$,

(5) $\sum_{i=1}^{N}\left\|q_{i}\right\|_{z}^{2} \geq \theta\|g\|_{z}^{2}$ for $z \in \partial \Omega$.

Proof Let $\gamma=\frac{1}{16 N_{1}^{2} N_{2}^{2}}$ where $N_{1}, N_{2} \in \mathbb{N}$ are constants from Theorems 1 and 2 respectively. We define polynomials $q_{1}, \ldots, q_{N}$ by induction so that properties $1-4$ are fulfilled. Let $q_{1}=0$. Suppose that we have just defined $q_{1}, \ldots, q_{k}$ so that (1)-(4) are fulfilled. Let

$$
h=\min \left\{a g, g-\left|\sum_{i=1}^{k} q_{i}\right|\right\} \text {. }
$$

In particular $h>0$ on $\partial \Omega$, so we can use Theorem 1 to obtain polynomials $f_{1}, \ldots, f_{N_{1}}$ such that:

- $\left|f_{i}\right|<\frac{h}{N_{1}}$ on $\partial \Omega$,

- $\frac{h}{2 N_{1}}<\max _{i=1, \ldots, N_{1}}\left|f_{i}\right|$.

Now for a given $j$ due to Theorem 2 we can choose homogeneous polynomials $p_{j, 1}, \ldots, p_{j, N_{2}}$ with degrees $m_{j, 1}, \ldots, m_{j, N_{2}}$ respectively so that

$$
\frac{1}{2}<\max _{i=1, \ldots, N_{2}}\left|p_{j, i}(z)\right|<1
$$

Let

$$
q_{k+1}:=\frac{1}{N_{2}} \sum_{j=1}^{N_{1}} \sum_{i=1}^{N_{2}} f_{j} p_{j, i}
$$

We can enlarge degrees if necessary so that polynomials $f_{j} p_{j, i}$ are orthogonal and $q_{k+1}$ fulfills property (1).

Since $h \leq a g$ we observe the property (2):

$$
\left|q_{k+1}\right| \leq \frac{1}{N_{2}} \sum_{j=1}^{N_{1}} \sum_{i=1}^{N_{2}}\left|f_{j}\right|\left|p_{j, i}\right|<\frac{1}{N_{2}} \sum_{j=1}^{N_{1}} \sum_{i=1}^{N_{2}} \frac{h}{N_{1}} \leq \frac{1}{N_{2}} \sum_{j=1}^{N_{1}} \sum_{i=1}^{N_{2}} \frac{a g}{N_{1}}=a g
$$

The property (3) is obvious:

$$
\begin{aligned}
\left|\sum_{i=1}^{k+1} q_{i}\right| & \leq\left|\sum_{i=1}^{k} q_{i}\right|+\left|q_{k+1}\right|<\left|\sum_{i=1}^{k} q_{i}\right|+\frac{1}{N_{2}} \sum_{j=1}^{N_{1}} \sum_{i=1}^{N_{2}} \frac{h}{N_{1}} \leq\left|\sum_{i=1}^{k} q_{i}\right|+h \\
& \leq\left|\sum_{i=1}^{k} q_{i}\right|+g-\left|\sum_{i=1}^{k} q_{i}\right|=g .
\end{aligned}
$$


Since $f_{j} p_{j, i}$ are orthogonal we can estimate for $z \in \partial \Omega$ :

$$
\begin{aligned}
\left\|q_{m+1}\right\|_{z}^{2} & =\frac{1}{N_{2}^{2}} \sum_{j=1}^{N_{1}} \sum_{i=1}^{N_{2}}\left\|f_{j} p_{j, i}\right\|_{z}^{2}=\frac{1}{N_{2}^{2}} \sum_{j=1}^{N_{1}}\left\|f_{j}\right\|_{z}^{2} \sum_{i=1}^{N_{2}}\left|p_{j, i}(z)\right| \geq \frac{1}{N_{2}^{2}} \sum_{j=1}^{N_{1}}\left\|f_{j}\right\|_{z}^{2} \frac{1}{4} \\
& \geq \frac{1}{4 N_{2}^{2}}\left\|\max _{i=1, \ldots, N_{1}}\left|f_{i}\right|\right\|_{z}^{2}>\frac{1}{16 N_{1}^{2} N_{2}^{2}}\|h\|_{z}^{2}=\gamma\|h\|_{z}^{2}
\end{aligned}
$$

which is the property (4) and finishes a construction of polynomials with required properties (1)-(4).

Let us choose polynomials $q_{1}, \ldots, q_{N}$ which fulfill properties (1)-(4). Let

$$
s_{N}: \partial \Omega \ni z \rightarrow\left\|\sum_{k=1}^{N} q_{k}\right\|_{z}^{2}=\sum_{k=1}^{N}\left\|q_{k}\right\|_{z}^{2} .
$$

We have defined an increased sequence of continuous bounded functions. For a given $z \in \partial \Omega$ there exists $\lim _{N \rightarrow \infty} s_{N}(z) \leq\|g\|_{z}^{2}$. In particular $\lim _{k \rightarrow \infty}\left\|q_{k}\right\|_{z}^{2}=0$, which implies that $\lim _{m \rightarrow \infty}\left\|g-\left|\sum_{i=1}^{m} q_{i}\right|\right\|_{z}=0$ and $\lim _{N \rightarrow \infty} s_{N}(z)=\|g\|_{z}^{2}$. Now, due to Dini's theorem $s_{N}$ is convergent uniformly to $z \rightarrow\|g\|_{z}^{2}$, so there exists $N \in \mathbb{N}$ such that $s_{N} \geq \theta\|g\|_{z}^{2}$ on $\partial \Omega$ [property (5)].

Theorem 4 There exists a holomorphic function $f \in A(\Omega)$ such that every slice function has a series of Taylor coefficients divergent with every power $s \in[0,2)$.

Proof For a given $j \in \mathbb{N}, g \equiv 1, a=2^{-j}$ and $\theta=0.5$ let us use Lemma 3 to obtain a number $N_{j}$ and polynomials $q_{j, 1}, \ldots, q_{j, N_{j}}$. We can assume that polynomials $q_{j, i}$ are chosen so that different polynomials contain monomials with different degrees.

Let us summarize all the necessary properties of polynomials $q_{j, i}$ :

(1) If $I(j, i)$ is a list of all degrees of monomials in homogeneous expansion of $q_{j, i}$ then $^{2} I(j, i)<I(j, i+1)$ and $I(j, *)<I(j+1, *)$.

(2) $\left|q_{j, i}\right|<2^{-j}$ on $\partial \Omega$,

(3) $\left|\sum_{i=1}^{N_{j}} q_{j, i}\right|<1$ on $\partial \Omega$,

(4) $\sum_{i=1}^{N_{j}}\left\|q_{j, i}\right\|_{z}^{2} \geq 0.5$ for $z \in \partial \Omega$.

Let us define

$$
f=\sum_{j=1}^{\infty} \frac{1}{j^{2}} \sum_{i=1}^{N_{j}} q_{j, i}
$$

Since $\left|\sum_{i=1}^{N_{j}} q_{j, i}\right|<1$, we just defined a holomorphic function which is continuous to the boundary $(f \in A(\Omega))$. Now for a given polynomial $q_{j, i}$ we have a homogeneous expansion:

\footnotetext{
${ }^{2}$ If $A, B$ are sets then $A<B \Leftrightarrow \forall x \in A, y \in B x<y$.
} 


$$
q_{j, i}=\sum_{k \in I(j, i)} p_{j, i, k}
$$

where $I(j, i)$ is a list of degrees of all homogeneous polynomials $p_{j, i, k}$ in $q_{j, i}$ and $p_{j, i, k}$ has a degree equal to $k$.

In particular, using Taylor coefficients, we obtain the expansion:

$$
f(\lambda z)=\sum_{j=1}^{\infty} \sum_{i=1}^{N_{j}} \sum_{k \in I(j, i)} \frac{p_{j, i, k}(z)}{j^{2}} \lambda^{k}=\sum_{j, i, k} \frac{p_{j, i, k}(z)}{j^{2}} \lambda^{k}
$$

for $z \in \partial \Omega$.

Let $s \in[0,2)$. The property (2) gives us $\left\|q_{j, i}\right\|_{z} 2^{j}<1$ for $z \in \partial \Omega$ and:

$$
\left\|q_{j, i}\right\|_{z}^{s} \geq\left\|q_{j, i}\right\|_{z}^{s}\left(\left\|q_{j, i}\right\|_{z} 2^{j}\right)^{2-s}=\left\|q_{j, i}\right\|_{z}^{2} 2^{j(2-s)} .
$$

Since $\left\|q_{j, i}\right\|_{z}^{2}=\sum_{k}\left\|p_{j, i, k}\right\|_{z}^{2}$ we have

$$
\left\|q_{j, i}\right\|_{z}^{s} \leq\left(\sum_{k}\left\|p_{j, i, k}\right\|_{z}^{2}\right)^{s / 2} \leq \sum_{k}\left\|p_{j, i, k}\right\|_{z}^{s}
$$

which together with the property (4) and inequality (2.1) gives us:

$$
\begin{aligned}
\sum_{j, i, k}\left|\frac{p_{j, i, k}(z)}{j^{2}}\right|^{s} & =\sum_{j, i, k} j^{-2 s}\left\|p_{j, i, k}\right\|_{z}^{s} \geq \sum_{j, i} j^{-2 s}\left\|q_{j, i}\right\|_{z}^{s} \\
& \geq \sum_{j, i} j^{-2 s}\left\|q_{j, i}\right\|_{z}^{2} 2^{j(2-s)} \geq \sum_{j} 2^{-1} j^{-2 s} 2^{j(2-s)}=\infty
\end{aligned}
$$

In other words any slice function $\lambda \rightarrow f(\lambda z)$ has a series of Taylor coefficients divergent with every power $s \in[0,2)$.

As a second application of Lemma 3 we give a construction of inner function:

Theorem 5 There exists an inner function $f \in \mathbb{O}(\Omega)$ such that for all $z \in \partial \Omega$ we have following properties:

- $\left|f_{z}^{*}\right|=1$ a.e. on $\partial \mathbb{D}$,

- $f_{z}$ has a series of Taylor coefficients divergent with every power $s \in[0,2)$.

Proof We construct a sequence of orthogonal polynomials $\left\{p_{j}\right\}$ with the following properties: 
(1) $\left|\sum_{j=1}^{m} p_{j}(z)\right|<1$ for $z \in \partial \Omega, m \geq 1$,

(2) $\left\|p_{m}\right\|_{z}^{2} \geq \frac{1}{2}\left\|1-\left|\sum_{j=1}^{m-1} p_{j}\right|\right\|_{z}^{2}$ for $z \in \partial \Omega, m>1$,

(3) $p_{j}$ is a sum of orthogonal polynomials: $p_{j}=\sum_{i=1}^{N_{j}} p_{j, i}$ with the following property:

$$
\left|p_{m, i}\right|<\left(1-\left|\sum_{j=1}^{m-1} p_{j}\right|\right)^{m}
$$

on $\partial \Omega, m>1$.

Let $N_{1}=1, p_{1}=p_{1,1} \equiv 0$. Properties (1)-(3) are fulfilled so let us assume that we have defined $p_{1}, \ldots, p_{m}$.

Due to Lemma 3 used for the data: $a=\inf _{z \in \partial \Omega}\left(1-\left|\sum_{j=1}^{m} p_{j}(z)\right|\right)^{m}, \theta=$ $\frac{1}{2}, g=1-\left|\sum_{j=1}^{m} p_{j}\right|$ we can choose number $N_{m+1} \in \mathbb{N}$ and orthogonal polynomials $p_{m+1,1}, \ldots, p_{m+1, N_{m+1}}$ with the following properties:

- $p_{1}, \ldots, p_{m+1}, \sum_{i=1}^{N_{m+1}} p_{m+1, i}$ are orthogonal,

- $\left|p_{m+1, i}\right|<a g \leq\left(1-\left|\sum_{j=1}^{m} p_{j}\right|\right)^{m+1}$ on $\partial \Omega$,

- $\left|\sum_{i=1}^{N_{m+1}} p_{m+1, i}\right|<g$ on $\partial \Omega$,

- $\left\|\sum_{i=1}^{N_{m+1}} p_{m+1, i}\right\|_{z}^{2} \geq \frac{1}{2}\|g\|_{z}^{2}$ for $z \in \partial \Omega$.

We can choose $p_{m+1}=\sum_{i=1}^{N_{m+1}} p_{m+1, i}$ and observe that properties (1)-(3) are fulfilled, which finishes the construction of a sequence $\left\{p_{j}\right\}$ by induction.

Now we can define:

$$
f=\sum_{j=1}^{\infty} p_{j}
$$

Since $\left\{p_{j}\right\}$ are orthogonal and $\left|\sum_{j=1}^{N} p_{j}\right|<1$ on $\partial \Omega$, we have: $\sum_{j=1}^{N}\left\|p_{j}\right\|_{z}^{2}<1$, which implies that: $\sum_{j=1}^{\infty}\left\|p_{j}\right\|_{z}^{2} \leq 1$ for $z \in \partial \Omega$. In particular $\lim _{j \rightarrow \infty}\left\|p_{j}\right\|_{z}=0$ for $z \in \partial \Omega$. The property (2) implies that $\lim _{N \rightarrow \infty}\left\|1-\left|\sum_{j=1}^{N} p_{j}\right|\right\|_{z}=0$ and $\sum_{j=1}^{\infty}\left\|p_{j}\right\|_{z}^{2}=1$ for $z \in \partial \Omega$. We can conclude that $f \in \mathbb{O}(\Omega),|f| \leq 1$ on $\partial \Omega$ and $\|f\|_{z}=1$ for $z \in \partial \Omega$. Let us note that every slice of $f$ is inner.

Now we estimate series of Taylor coefficients. For a given polynomial $p_{j, i}$ we have the homogeneous expansion:

$$
p_{j, i}=\sum_{k \in I(j, i)} p_{j, i, k}
$$


where $I(j, i)$ is a list of degrees of all homogeneous polynomials $p_{j, i, k}$ in $p_{j, i}$ and $p_{j, i, k}$ has a degree equal to $k$. Now, using Taylor coefficients, we have the following expansion:

$$
f(\lambda z)=\sum_{j=1}^{\infty} \sum_{i=1}^{N_{j}} \sum_{k \in I(j, i)} p_{j, i, k}(z) \lambda^{k}=\sum_{j, i, k} p_{j, i, k}(z) \lambda^{k}
$$

for $z \in \partial \Omega$.

Let $s \in[0,2)$. The property (3) gives us $\left\|p_{m, i}\right\|_{z}\left\|1-\left|\sum_{j=1}^{m-1} p_{j}\right|\right\|_{z}^{-m}<1$ and implies:

$$
\begin{aligned}
\left\|p_{m, i}\right\|_{z}^{s} & \geq\left\|p_{m, i}\right\|_{z}^{s}\left(\left\|p_{m, i}\right\|_{z}\left\|1-\left|\sum_{j=1}^{m-1} p_{j}\right|\right\|_{z}^{-m}\right)^{2-s} \\
& =\left\|p_{m, i}\right\|_{z}^{2}\left\|1-\left|\sum_{j=1}^{m-1} p_{j}\right|\right\|_{z}^{m(s-2)}
\end{aligned}
$$

for $z \in \partial \Omega, m>1$.

Since $\left\|p_{j, i}\right\|_{z}^{2}=\sum_{k}\left\|p_{j, i, k}\right\|_{z}^{2}$ we have

$$
\left\|p_{j, i}\right\|_{z}^{s} \leq\left(\sum_{k}\left\|p_{j, i, k}\right\|_{z}^{2}\right)^{s / 2} \leq \sum_{k}\left\|p_{j, i, k}\right\|_{z}^{s}
$$

which together with the property (2) and inequality (2.2) gives us:

$$
\begin{aligned}
\sum_{j, i, k}\left|p_{j, i, k}(z)\right|^{s} & =\sum_{j, i, k}\left\|p_{j, i, k}\right\|_{z}^{s} \geq \sum_{j, i}\left\|p_{j, i}\right\|_{z}^{s} \geq \sum_{m>1, i}\left\|p_{m, i}\right\|_{z}^{2}\left\|1-\left|\sum_{j=1}^{m-1} p_{j}\right|\right\|_{z}^{m(s-2)} \\
& \geq \sum_{m>1}\left\|p_{m}\right\|_{z}^{2}\left\|1-\left|\sum_{j=1}^{m-1} p_{j}\right|\right\|_{z}^{m(s-2)} \geq \sum_{m>1} \frac{1}{2}\left\|1-\left|\sum_{j=1}^{m-1} p_{j}\right|\right\|_{z}^{2+m(s-2)} \\
& \geq \sum_{m>\frac{2}{2-s}} \frac{1}{2}=\infty
\end{aligned}
$$

In other words, any slice function $\lambda \rightarrow f(\lambda z)$ has a series of Taylor coefficients divergent with every power $s \in[0,2)$.

Now let us improve the last Theorem: 
Theorem 6 Let $\psi$ be a positive LSC function on $\partial \Omega$, whose every slice function $\psi_{z}: \partial \mathbb{D} \ni \lambda \rightarrow \psi(\lambda z)$ is from $L^{2}$. There exists a holomorphic function $f \in \mathbb{O}(\Omega)$ such that for all $z \in \partial \Omega$ we have the following properties:

- $\left|f_{z}^{*}\right|=\psi_{z}$ a.e. on $\partial \mathbb{D}$,

- $f_{z}$ has a series of Taylor coefficients divergent with every power $s \in[0,2)$.

Proof There exists a sequence of positive, continuous functions $\left\{\psi_{k}\right\}$ such that (pointwise):

$$
0<\psi_{1}<\cdots<\psi_{n}<\psi_{n+1}<\cdots \leq \lim _{n \rightarrow \infty} \psi_{n}=\psi
$$

Let $\phi_{1}=\psi_{1}$. We construct a sequence of orthogonal polynomials $\left\{p_{j}\right\}$ and continuous, positive functions $(m>1)$ :

$$
\phi_{m}: \partial \Omega \ni z \rightarrow \min \left\{\psi_{m}(z),\left|\sum_{j=1}^{m-1} p_{j}(z)\right|+1\right\}
$$

with the following properties:

(1) $\left|\sum_{j=1}^{m} p_{j}\right|<\phi_{m}$ on $\partial \Omega, m \geq 1$,

(2) $\left|\sum_{j=1}^{m-1} p_{j}\right|<\phi_{m}$ on $\partial \Omega, m>1$,

(3) $\left\|p_{m}\right\|_{z}^{2} \geq \frac{1}{2}\left\|\phi_{m}-\left|\sum_{j=1}^{m-1} p_{j}\right|\right\|_{z}^{2}$ for $z \in \partial \Omega, m>1$,

(4) $p_{j}$ is a sum of orthogonal polynomials: $p_{j}=\sum_{i=1}^{N_{j}} p_{j, i}$ with: $\left|p_{m, i}\right|<$ $\left(\phi_{m}-\left|\sum_{j=1}^{m-1} p_{j}\right|\right)^{m}$ on $\partial \Omega, m>1$.

Let $N_{1}=1, p_{1}=p_{1,1} \equiv 0$. The properties (1)-(4) are fulfilled for $m=1$ so let us assume that we have defined $p_{1}, \ldots, p_{m}$ with the properties (1)-(4).

Let us observe inequalities $\left|\sum_{j=1}^{m} p_{j}\right|<\phi_{m} \leq \psi_{m}<\psi_{m+1}$, which gives us the property (2): $\left|\sum_{j=1}^{m} p_{j}\right|<\phi_{m+1}$ on $\partial \Omega$ so we can use Lemma 3 for the data:

$$
a=\inf _{\partial \Omega}\left(\phi_{m+1}-\left|\sum_{j=1}^{m} p_{j}\right|\right)^{m}, \theta=\frac{1}{2}, g=\phi_{m+1}-\left|\sum_{j=1}^{m} p_{j}\right|
$$

and we can obtain a number $N_{m+1} \in \mathbb{N}$ and polynomial $p_{m+1}:=\sum_{i=1}^{N_{m+1}} p_{m+1, i}$ where $p_{m+1,1}, \ldots, p_{m+1, N_{m+1}}$ are orthogonal polynomials with the following properties:

- $p_{1}, \ldots, p_{m+1}, p_{m+1,1}, \ldots, p_{m+1, N_{m+1}}$ are orthogonal,

- $\left|p_{m+1, i}\right|<a g \leq\left(\phi_{m+1}-\left|\sum_{j=1}^{m} p_{j}\right|\right)^{m+1}$ on $\partial \Omega$, (the property 4 ), 
- $\left|p_{m+1}\right|<g=\phi_{m+1}-\left|\sum_{j=1}^{m} p_{j}\right|$ on $\partial \Omega$,

- $\left\|p_{m+1}\right\|_{z}^{2} \geq \frac{1}{2}\|g\|_{z}^{2}=\frac{1}{2}\left\|\phi_{m+1}-\left|\sum_{j=1}^{m} p_{j}\right|\right\|_{z}^{2}$ for $z \in \partial \Omega$, (the property 3).

We can easily observe the property (1):

$$
\left|\sum_{j=1}^{m+1} p_{j}\right| \leq\left|p_{m+1}\right|+\left|\sum_{j=1}^{m} p_{j}\right|<g+\left|\sum_{j=1}^{m} p_{j}\right|=\phi_{m+1} .
$$

Now the properties (1)-(4) are fulfilled, which finishes the construction of the sequence $\left\{p_{j}\right\}$ by induction.

Let us define

$$
f=\sum_{j=1}^{\infty} p_{j}
$$

Since $\left\{p_{j}\right\}$ are orthogonal and $\left|\sum_{j=1}^{m} p_{j}\right|<\phi_{m} \leq \psi_{m} \leq \psi$ on $\partial \Omega$ we have: $\sum_{j=1}^{m}\left\|p_{j}\right\|_{z}^{2}<\|\psi\|_{z}^{2}<\infty$, which implies that: $\sum_{j=1}^{\infty}\left\|p_{j}\right\|_{z}^{2} \leq\|\psi\|_{z}^{2}$ for $z \in \partial \Omega$. In particular $\lim _{j \rightarrow \infty}\left\|p_{j}\right\|_{z}=0$ and $\lim _{m \rightarrow \infty}\left\|\phi_{m}-\left|\sum_{j=1}^{m-1} p_{j}\right|\right\|_{z}=0$ for $z \in \partial \Omega$.

Since $\phi_{m} \leq \psi$ and $\|\psi\|_{z}<\infty$ we can conclude that $f_{z} \in H^{2}(\mathbb{D})$ so there exists $f_{z}^{*}$ a.e. on $\partial \mathbb{D}$ for $z \in \partial \Omega$. Suppose that there exists $\delta>0$ and $z \in \partial \Omega$ such that $\left\|\psi-\left|f^{*}\right|\right\|_{z}>3 \delta>0$. In particular, there exists $\delta_{2}>0$ and a set $A \subset \partial \mathbb{D}$ with a measure $\mu(A)>\delta_{2}$ such that there exist $f_{z}^{*}$ on $A$ and $\psi(\lambda z)-\left|f^{*}(\lambda z)\right|>2 \delta$ for $\lambda \in A$. Since $\lim _{m \rightarrow \infty}\left\|\phi_{m}-\left|\sum_{j=1}^{m-1} p_{j}\right|\right\|_{z}=0$ we have $\lim _{m \rightarrow \infty}\left\|\phi_{m}-\left|f^{*}\right|\right\|_{z}=0$, so decreasing a little $A$ if necessary, we can assume that $\mu(A)>\delta_{2}$ and there exists $m_{0} \in \mathbb{N}$ so large that $\psi_{m}-2 \delta>\left|f^{*}\right| \geq \phi_{m}-\delta$ on $A z$ for $m \geq m_{0}$. In particular $\psi_{m}>\phi_{m}+\delta$ on $A z$ for $m \geq m_{0}$, which implies that $\phi_{m}:=\left|\sum_{j=1}^{m-1} p_{j}\right|+1$ on $A z$ for $m \geq m_{0}$. But

$$
\left\|p_{m}\right\|_{z}^{2} \geq \frac{1}{2}\left\|\phi_{m}-\left|\sum_{j=1}^{m-1} p_{j}\right|\right\|_{z}^{2} \geq \frac{1}{2} \int_{A z}\left(\phi_{m}-\left|\sum_{j=1}^{m-1} p_{j}\right|\right)^{2} d \mu=\frac{1}{2} \mu(A)>\frac{1}{2} \delta_{2}
$$

for $m \geq m_{0}$ which is impossible because, as we mentioned, $\lim _{j \rightarrow \infty}\left\|p_{j}\right\|_{z}=0$ for $z \in \partial \Omega$. In particular $\left\|\psi-\left|f^{*}\right|\right\|_{z}=0$ for $z \in \partial \Omega$ i.e. $\left|f_{z}^{*}\right|=\psi_{z}$ a.e. on $\partial \mathbb{D}$.

Now we estimate a series of Taylor coefficients. For a given polynomial $p_{j, i}$ we have the homogeneous expansion:

$$
p_{j, i}=\sum_{k \in I(j, i)} p_{j, i, k}
$$


where $I(j, i)$ is a list of degrees of all homogeneous polynomials $p_{j, i, k}$ in $p_{j, i}$ and $p_{j, i, k}$ has a degree equal to $k$, which, using Taylor coefficients, gives us the expansion:

$$
f(\lambda z)=\sum_{j=1}^{\infty} \sum_{i=1}^{N_{j}} \sum_{k \in I(j, i)} p_{j, i, k}(z) \lambda^{k}=\sum_{j, i, k} p_{j, i, k}(z) \lambda^{k}
$$

for $z \in \partial \Omega$.

Let $s \in[0,2)$. The property (4) gives us $\left\|p_{m, i}\right\|_{z}\left\|\phi_{m}-\left|\sum_{j=1}^{m-1} p_{j}\right|\right\|_{z}^{-m}<1$ and implies:

$$
\left\|p_{m, i}\right\|_{z}^{s} \geq\left\|p_{m, i}\right\|_{z}^{s}\left(\left\|p_{m, i}\right\|_{z}\left\|\phi_{m}-\left|\sum_{j=1}^{m-1} p_{j}\right|\right\|_{z}^{-m}\right)^{2-s}=\left\|p_{m, i}\right\|_{z}^{2}\left\|\phi_{m}-\left|\sum_{j=1}^{m-1} p_{j}\right|\right\|_{z}^{m(s-2)}
$$

for $z \in \partial \Omega, m>1$.

Since $\left\|p_{j, i}\right\|_{z}^{2}=\sum_{k}\left\|p_{j, i, k}\right\|_{z}^{2}$ we have

$$
\left\|p_{j, i}\right\|_{z}^{s} \leq\left(\sum_{k}\left\|p_{j, i, k}\right\|_{z}^{2}\right)^{s / 2} \leq \sum_{k}\left\|p_{j, i, k}\right\|_{z}^{s}
$$

which together with $0<\phi_{m}-\left|\sum_{j=1}^{m-1} p_{j}\right| \leq 1$ on $\partial \Omega$, the property (3) and inequality (2.3) gives us:

$$
\begin{aligned}
\sum_{j, i, k}\left|p_{j, i, k}(z)\right|^{s} & =\sum_{j, i, k}\left\|p_{j, i, k}\right\|_{z}^{s} \geq \sum_{j, i}\left\|p_{j, i}\right\|_{z}^{s} \geq \sum_{m>1, i}\left\|p_{m, i}\right\|_{z}^{2}\left\|\phi_{m}-\left|\sum_{j=1}^{m-1} p_{j}\right|\right\| \|_{z}^{m(s-2)} \\
& \geq \sum_{m>1}\left\|p_{m}\right\|_{z}^{2}\left\|\phi_{m}-\left|\sum_{j=1}^{m-1} p_{j}\right|\right\|_{z}^{m(s-2)} \geq \sum_{m>1} \frac{1}{2}\left\|\phi_{m}-\left|\sum_{j=1}^{m-1} p_{j}\right|\right\|_{z}^{2+m(s-2)} \\
& \geq \sum_{m>\frac{2}{2-s}} \frac{1}{2}=\infty
\end{aligned}
$$

In other words any slice function $\lambda \rightarrow f(\lambda z)$ has a series of Taylor coefficients divergent with every power $s \in[0,2)$.

Open Access This article is distributed under the terms of the Creative Commons Attribution 4.0 International License (http://creativecommons.org/licenses/by/4.0/), which permits unrestricted use, distribution, and reproduction in any medium, provided you give appropriate credit to the original author(s) and the source, provide a link to the Creative Commons license, and indicate if changes were made.

\section{References}

1. Dupain, Y.: Fonctions intérieures dans la boule unité de $\mathrm{Cn}$ dont les fonctions traces sont aussi intérieures. Math. Z. 198(2), 191-206 (1988) 
2. Fournier, J.: An interpolation problem for coefficients of $H_{\infty}$ function. Proc. Am. Math. Soc. 42, 402-408 (1972)

3. Kot, P.: A holomorphic function with given almost all boundary values on a domain with holomorphic support function. J. Convex Anal. 14(4), 693-704 (2007)

4. Kot, P.: Homogeneous polynomials on strictly convex domains. Proc. Am. Math. Soc. 135, 3895-3903 (2007)

5. Kot, P.: Bounded holomorphic functions with given maximal modulus on all circles. Proc. Am. Math. Soc. 137, 179-187 (2009)

6. Kot, P.: About boundary values in $A(\Omega)$. Trans. Am. Math. Soc. 363(8), 4063-4079 (2011)

7. Pełczyński, A.: Banach spaces of analytic functions and absolutely summing operators In: CBMS Regional Conference Series, Vol. 30. American Mathematical Society, Providence, (1977)

8. Rudin, W.: Function theory in the unit ball of $C^{n}$. Springer, New York (1980)

9. Ryll, J., Wojtaszczyk, P.: On homogeneous polynomials on a complex ball. Trans. Am. Math. Soc. 276, 107-116 (1983)

10. Wojtaszczyk, P.: On functions in the ball algebra. Proc. Am. Math. Soc. 85(2), 184-186 (1982)

11. Wojtaszczyk, P.: On values of homogeneous polynomials in discrete sets of points. Studia Math. 84, 97-104 (1986) 